\title{
Dimensión Vertical de la Región Anterior de la Mandíbula
}

\author{
Vertical Dimension in the Anterior Region of the Mandible \\ Paulo Domingos André Bolini; Ana Maria Minarelli Gaspar \& Juliana Feltrin de Souza
}

BOLINI, P. D. A.; GASPAR, M.A. M. \& DE SOUZA, J. F. Dimensión vertical de la región anterior de la mandíbula. Int. J. Morphol., 24(4):531-533, 2006.

RESUMEN: Realizamos mediciones de la región de la sínfisis mentoniana, en 308 mandíbulas secas: 247 pertenecientes a la colección del museo de cráneos de disciplina de Anatomía de la UNIFESP- Escuela Paulista de Medicina- EPM y 61 pertenecientes al laboratorio didáctico de la disciplina de Anatomía de la Facultad de Odontología de Araraquara- UNESP. En las mandíbulas dentadas, el mayor valor encontrado fue de 43,46 mm y el menor de 22,67 mm. En las mandíbulas desdentadas, el mayor valor encontrado fue de $37,24 \mathrm{~mm}$ y el menor de 6,22 mm.Esperamos poder contribuir para el mejor conocimiento de la región de la sínfisis mentoniana, tanto para los profesionales de las áreas de Cirugía e Implantología, como para los industriales de áreas afines, para la confección de instrumentales utilizados en estas prácticas.

PALABRAS CLAVE: Anatomía; Mandíbula; Sínfisis mentoniana.

\section{INTRODUCCIÓN}

La mandíbula, único hueso móvil de la cara, se localiza en el tercio inferior de la misma, se presenta en forma de herradura y junto al hueso hioides, forman el arco de fijación de los músculos del suelo bucal.

Castro (1985) describe a la mandíbula (maxilar inferior) como un hueso impar, en el cual se implantan los dientes inferiores, que se articulan, por las dos extremidades, con los huesos temporales correspondientes. Afirma que en la región anterior, de cada lado de la línea media, se encuentra un orificio, que corresponde foramen mentoniano, situado más frecuentemente en relación con el segundo premolar inferior.

Para Gardner et al. (1988), la superficie externa del cuerpo de la mandíbula es generalmente caracterizada por una cresta media, poco desarrollada que marca la línea de fusión de las dos mitades de la mandíbula, conocida como sínfisis mentoniana. Esto fue repetido por Costa (2000) quien afirma que corresponde a la región ántero- lateral de la mandíbula.

Fehrenbach \& Herring (1998) afirman que la fuerte región horizontal de la mandíbula, inferior al forámen mentoniano, es el cuerpo de la mandíbula, que presenta superiormente la parte alveolar y contiene las raíces de los dientes inferiores.
Según Okeson (2000), la mandíbula es sostenida por debajo del maxilar, por músculos, ligamentos y otros tejidos blandos, los cuales posibilitan la movilidad necesaria para la función con el maxilar.

Teixeira et al. (2001) relatan que el cuerpo de la mandíbula tiene la forma de herradura, y posee una fase externa y otra interna, limitadas por las márgenes superior e inferior, siendo que la fase externa presenta, en la región anterior, la sínfisis mentoniana, región media que representa la línea de fusión de las dos mitades del hueso fetal.

El manoseo rutinario de cráneos en las aulas prácticas de anatomía, nos permite observar grandes diferencias en la dimensión vertical de las mandíbulas en la región de la sínfisis mentoniana.

El proceso alveolar existe en función de los dientes, alcanzando su pleno desarrollo cuando todos los dientes permanentes ocupan sus lugares en la arcada dentaria, involucionando con la pérdida de los elementos dentales, hasta transformarse en un poco marcado reborde alveolar.

Realizamos el presente trabajo en mandíbulas con el objetivo de contribuir al el conocimiento anátomo-clínico-quirúrgico, que podrá ser de ayuda, a posibles formas de rehabilitación de los pacientes. 


\section{MATERIAL Y MÉTODO}

Fueron utilizadas 308 mandíbulas, 247 pertenecientes al museo de cráneos de la Disciplina de Anatomía de la UNIFESP- Escuela Paulista de Medicina- EPM y 61 al Laboratorio Didáctico de la disciplina de Anatomía de la Facultad de Odontología de Araraquara- UNESP, Brasil.

Fue utilizado un paquímetro digital Brown \& Sharpe, modelo EDP51766.

Las medidas fueron realizadas posicionando las puntas del paquímetro en la base de la mandíbula, en el sitio correspondiente al punto gnation (punto más inferior de la mandíbula en la línea media anterior) y en el punto interdentario inferior (punto mediano en la punta del proceso alveolar inferior, entre los incisivos centrales inferiores en el humano se usa la punta de la papila interdentaria entre los incisivos centrales inferiores). En las mandíbulas desdentadas se tomó el punto más alto del reborde alveolar correspondiente.

\section{RESULTADOS}

En base a las medidas realizadas en las mandíbulas, pertenecientes a la colección del museo de cráneos de la Disciplina de Anatomía de la Escuela Paulista de Medicina y de la Facultad de Odontología de Araraquara- UNESP, Brasil obtuvimos los siguientes datos, siendo el valor medio de todas las mandíbulas medidas igual a $30.50 \mathrm{~mm}$ :

Tabla I. Dimensión vertical de la región anterior en mandíbulas dentadas pertenecientes a la colección del museo de cráneos de la disciplina de Anatomía de la Escuela Paulista de Medicina y de la Facultad de Odontología de Araraquara- UNESP, Brasil.

\begin{tabular}{lr}
\hline Promedio & 32,63 \\
Mediana & 32,95 \\
Desviación Estándar & 4,29 \\
Valor mínimo & 22,67 \\
Valor máximo & 43,46 \\
\hline
\end{tabular}

Tabla II. Dimensión vertical de la región anterior en mandíbulas desdentadas, pertenecientes a la colección del museo de cráneos de la Disciplina de Anatomía de la Escuela Paulista de Medicina y de la facultad de Odontología de Araraquara- UNESP, Brasil.

\begin{tabular}{lr}
\hline Promedio & 24,97 \\
Mediana & 25,72 \\
Desviación Estándar & 6,41 \\
Valor mínimo & 6,22 \\
Valor máximo & 37,24 \\
\hline
\end{tabular}

\section{DISCUSIÓN}

La descripción más minuciosa de la mandíbula que fue posible encontrar correspondió a la de Testut \& Latarjet (1951) no habiendo referencias a las medidas de la dimensión vertical de la sínfisis mandibular.

Autores como Thomas (1989), Snodell et al. (1993) y Aki et al. (1994) estudiaron la región anterior de la mandíbula sobre una perspectiva ortodóncica y correlacionaron el tamaño y forma de la región de la sínfisis mentoniana con el diagnóstico de los pacientes ortodóncicos.

Campos et al. (1996), estudiando 50 mandíbulas humanas, secas, dentadas, sin deformidades, pertenecientes a individuos adultos brasileños de sexo, edad y raza desconocidos encontraron para la altura mentoniana, una media de 23,39 mm, con máxima de 32,20 mm y mínima de 12,90 mm.

Warwick \& Williams (1996) presentaron una minuciosa descripción, sin presentar valores métricos.

Toledo et al., (1998) realizaron un trabajo de medidas en hemi-mandíbulas maceradas e íntegras, de desdentados brasileños adultos, de sexo y grupos étnicos desconocidos, en 8 sitios padronizados, excepto la sínfisis mentoniana.

Varios trabajos acerca de medidas pueden ser encontrados en la área de ortodoncia, como el de Borrero et al. (1991) que relacionaron medidas angulares con crecimiento facial.

Vasconcellos \& Lindenblatt (2002), en los datos morfométricos de la sínfisis mandibular, presentan una media de $27.21 \mathrm{~mm}$ para la distancia en línea recta entre los bordes alveolar y basilar de la sínfisis mandibular y, valores máximo de $38.30 \mathrm{~mm}$ y mínimo de $17.62 \mathrm{~mm}$.

La media de las medidas encontradas por nosotros es de $30,50 \mathrm{~mm}$, mayor que la relatada por Vasconcellos \& Lindenblatt (2002) quienes encontraron una media de $27,21 \mathrm{~mm}$, también mayor que la obtenida por Campos, que fue de 23,39 mm; esta última, que es baja, se explica porque las mandíbulas son edentadas. Nuestra mayor medida fue de 43,46, valor mayor que el obtenido por Vasconcellos \& Lindenblatt, que fue de $38,30 \mathrm{~mm}$, mayor también que el descrito por Campos, correspondiente a 32,20 mm. El menor valor que encontramos fue de 6.22, menor valor que el informado por Vasconcellos \& Lindenblatt de 17.62, también menor que el encontrado por Campos, que fue de 12.90 $\mathrm{mm}$. Creemos que tal variación se debe al mayor número de mandíbulas nuestra muestra. 
A partir de los análisis descriptivo y cuantitativo, nuestro trabajo nos permite, conforme previsto, observar que las mandíbulas desdentadas presentan una dimensión vertical anterior menor a la media encontrada en las mandíbulas dentadas.
Esperamos que con nuestros resultados podamos contribuir con los profesionales de las áreas de Cirugía e Implantología, así como con los industriales de áreas afines para la confección de instrumentales utilizados en estas prácticas.

BOLINI, P. D. A.; GASPAR, M. A. M. \& DE SOUZA, J. F. Vertical dimension in the anterior region of the mandible. Int. J. Morphol., 24(4):531-533, 2006.

SUMMARY: We made some measurements from the region of the symphysis menti in 308 dried mandibles; 247 belong to the collection of the cranial museum of the discipline of Anatomy of the UNIFESP- Escola Paulista de MedicinaEPM and 61 belong to the didactic laboratory of the discipline of Anatomy from the Faculdade de Odontologia de AraraquaraUNESP, Brasil. On the dentate mandibles, the highest value founded was of 43,46 mm and the lowest of 22,67 mm. On the edentate mandibles, the highest value founded was of $37,24 \mathrm{~mm}$ and the lowest of $6,22 \mathrm{~mm}$. We hope that with the following discoveries we could contribute to a better knowledge of the symphysis menti region, not only to the professionals in Surgery and Implantology areas, but the industrials of related areas, to the confection of instruments used in these practices.

KEY WORDS: Anatomy; Mandible; Symphysis menti.

\section{REFERENCIAS BIBLIOGRÁFICAS}

Aki, T; Nanda, R. S.; Currier, G. F. \& Nanda, S. K. Assessment of symphysis morphology as a predictor of the direction of mandibular growth. Am. J. Orthod. Dentofacial Orthopedics, 106(1):60-9, 1994.

Borrero, P.; O`Reilly, M. T. \& Close, J. Relação entre dimensão vertical esquelética, contorno mandibular e aumento do comprimento mandibular em indivíduos masculinos. Rev. Paul. Odontol. 13(2):25-33, 1991.

Campos, A. E. S.; Vasconcellos, H. A. de \& Barbosa, C. Mandíbulas Adultas Humanas: Alterações Morfológicas. 53(4-6): 52-4, 1996.

Castro, S. V de. Anatomia Fundamental. 2a . ed. São Paulo, Makron Books, 1985.

Costa, C. Fundamentos de Anatomia para o Estudante de Odontologia. São Paulo, Atheneu, 2000.

Fehrenbach, M. J. \& Herring, S. H. Anatomia Ilustrada da Cabeça e do Pescoço. São Paulo, Manole, 1998.

Gardner, E.; Gray, D. J. \& O’Rahilly, R. Anatomía. Estudo do corpo humano. $4^{\mathrm{a}}$ ed. Rio de Janeiro, Guanabara Koogan, 1988.

Okeson, J. P. Tratamento das desordens temporomandibulares e oclusão. $4^{\text {a }}$ ed. São Paulo, Artes Médicas, 2000.
Snodell, S. F.; Nanda, R. S. \& Currier, G. F. A longitudinal cephalometric study of transferense and vertical craniofacial growth. Am J. Orthod. and Dentofacial Orthopedics, 1:471-83, 1993.

Teixeira, L. M. S.; Reher, P. \& Reher, V. G. S. Anatomia Aplicada à Odontologia. Rio de Janeiro, Guanabara Koogan, 2001.

Testut, L. \& Latarjet, A. Tratado de Anatomia Humana, Barcelona, Salvat, 1951.

Thomas, H. Research on predictability in cephalometry of the relationship of chin symphysis and nose-chin balance. Am. J. Orthod. and Dentofacial Orthopedics., 96 (2):110-9, 1989

Toledo Filho, J. L.; Marzola, C. \& Toledo Neto, J. L. Estudo Morfométrico Seccional da Mandíbula Aplicado às Técnicas de Implantodontia, Cirurgia e Traumatologia Bucomaxilofacial. Rev. FOB, 6(1):23-39, 1998.

Vasconcellos, H. A. de; Lindenblatt, R. de C. Morfologia e morfometria da sínfise mandibular: aplicação prática na Implantodontia. RBO, 59(5):332-4, 2002.

Warwick, R. \& Williams, P. L. Gray Anatomia. Rio de Janeiro, Guanabara Koogan, 1995.

Dirección para correspondencia:

Prof. Dr. Paulo Domingos André Bolini

Faculdade de Odontologia de Araraquara- ULNESP

Departamento de Morfologia

Rua Humaitá 1680

Araraquara, SP - 14801-903

BRASIL

Recibido : 24-04-2006 Aceptado: 22-08-2006 
